\title{
Erratum to: A Classification of Conjugately Separated Abelian, Commutative Transitive and Restricted Gromov One-Relator Groups
}

Benjamin Fine, Alexei Myasnikov, Volkmar große Rebel and Gerhard Rosenberger

Erratum to: Results. Math. (2007) 50:183-193

DOI 10.1007/s00025-007-0245-5

The following are typos which obscure parts of the proof.

(1) Page 186 line 5 should read " $\left[x, y^{2}\right]=\left[y^{2}, y\right]=1$ "

(2) Page 188 line 12 should read "If $\beta \geq 2$ then $\left\langle z^{\alpha}, z w\right\rangle$ is..."

(3) Page 189 line 9 from the bottom should read "If $|\alpha| \geq 2$ then $\left\langle x^{\alpha}, x y x y^{-1}\right\rangle$ is..."

Benjamin Fine

Department of Mathematics

Fairfield University

Fairfield, Connecticut 06430

USA

e-mail: fine@fair1.fairfield.edu

Alexei Myasnikov

Department of Mathematics and Statistics

McGill University

Montreal, QC H3A 2K6

Canada

e-mail: alexeim@math.mcgill.ca

The online version of the original article can be found under doi:10.1007/s00025-007-0245-5. 
Volkmar große Rebel and Gerhard Rosenberger

Universität Dortmund

Fachbereich Mathematik

44221 Dortmund

Germany

e-mail: volkmar.rebel@math.uni-dortmund.de;

gerhard.rosenberger@math.uni-dortmund.de 